J. Lake Sci. (湖泊科学), 2020, 32(4): 1041-1049

DOI 10. 18307/2020. 0413

(c) 2020 by Journal of Lake Sciences

\title{
针铁矿对湖泊草、藻来源可溶有机质的非均质吸附"
}

\author{
章 奇 $^{1}$, 居 琪 $^{1}$, 李健欣 ${ }^{2}$, 曹驰程 ${ }^{1}$, 江和龙 ${ }^{3}$, 张 晖 $^{1 * *}$ \\ ( 1 : 东南大学公共卫生学院,环境科学工程教育部重点实验室,南京 210009) \\ (2: 南京林业大学生物与环境学院, 江苏省环境工程重点实验室,南京 210037) \\ (3: 中国科学院南京地理与湖泊研究所,湖泊与环境国家重点实验室,南京 210008)
}

\begin{abstract}
摘 要: 为探究富营养化湖泊中自生源可溶有机质 (DOM) 在泥水界面的吸附行为, 以马来眼子菜 (Potamogeton malaianus) 和铜绿微囊藻 (Microcystis aeruginosa) 释放的 DOM 为代表, 考察针铁矿对草、藻源 DOM 中不同组分的吸附特 征. 三维菼光平行因子分析表明类富里酸组分 $\mathrm{C} 1$ 和类胡敏酸组分 $\mathrm{C} 4$ 的含量很低, 而类蛋白物质 (类酪氨酸组分 $\mathrm{C} 2$ 和类 色氨酸组分 C3) 分别占草、藻源 DOM 苂光组成的 70\%和 $93 \% .2$ 种 DOM 均可被针铁矿吸附, 吸附过程符合伪一级动力 学. 通过等温线拟合发现针铁矿吸附藻源 DOM 的非线性更强, 饱和吸附量 $(23.77 \mathrm{mg} / \mathrm{g})$ 高于草源 DOM $(19.10 \mathrm{mg} / \mathrm{g})$. 特 别地, 类蛋白组分呈现非线性吸附, 而类腐殖物质的吸附近似线性, 且针铁矿对于 DOM 各苂光组分的吸附量顺序为: C3 > $\mathrm{C} 2>\mathrm{C} 4>\mathrm{C} 1$, 此非均质吸附特征与 DOM 组分的初始含量、分子大小、芳香性及有效吸附位点有关. 红外光谱证实氨基、 羧基和羟基是吸附过程中的重要官能团. 因此, 草、藻源 DOM 显著改变了针铁矿表面的有机物质组成, 影响湖泊沉积物 的生物地球化学行为.
\end{abstract}

关键词: 可溶有机质; 针铁矿; 吸附; 平行因子分析;马来眼子菜; 铜绿微囊藻

\section{Heterogeneous adsorption of macrophyte- and algae-derived dissolved organic matter on goethite in freshwater lakes*}

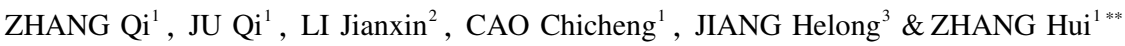 \\ (1: Key Laboratory of Environmental Medicine Engineering, Ministry of Education, School of Public Health, Southeast Uni- \\ versity, Nanjing 210009, P.R.China) \\ (2: Key Laboratory of Environmental Engineering of Jiangsu Province, College of Biology and the Environment, Nanjing For- \\ estry University, Nanjing 210037, P.R.China) \\ (3: State Key Laboratory of Lake Science and Environment, Nanjing Institute of Geography and Limnology, Chinese Academy \\ of Sciences, Nanjing 210008, P.R.China)
}

Abstract: Adsorptive fractionation of macrophyte- and algae-derived dissolved organic matter (MDOM and ADOM) on goethite was investigated to demonstrate the transfer of autochthonous DOM at the water-sediment interface in eutrophic lakes. Excitation emission matrix spectra with parallel factor analysis showed that protein-like substances, including tyrosine- and tryptophan-like component ( C2 and C3), accounted for 70\% and 93\% of total fluorescent components in MDOM and ADOM, respectively. While, contents of humic acid- and fulvic acid-like components ( C4 and C1) were limited in both DOMs. The two DOMs were effectively adsorbed on goethite and this adsorption process can be well simulated by pseudo-first order kinetic equation. Fitting by adsorption isotherm revealed the stronger, more nonlinear adsorption of ADOM $\left(23.77 \mathrm{mg} / \mathrm{g}\right.$ of $\left.Q_{\max }\right)$ on goethite than MDOM (19.10 mg/g of $\left.Q_{\max }\right)$. Specifically, a nonlinear adsorption was found between protein-like substances and goethite, whereas the adsorption of humic-like substances was almost linear. The adsorption capacity of DOM components to goethite followed the order of: C3 > C2 > C4 > C1, which was dependent on their initial content, molecular weights, aromaticity, and binding sites. Infrared spectroscopy further indicated that amino, carboxyl, and hydroxyl were responsible functional groups for the heterogeneous adsorption. Overall,

* 2019-07-03 收稿; 2020-01-08 收修改稿.

东南大学通识课程项目 (1125000162) 和中央高校教育教学改革专项资金项目 (5225009110) 联合资助.

** 通信作者; E-mail: 13851553232@163.com. 
the production of ADOM and MDOM significantly changes the organic compositions on goethite surface and affects the biogeochemical cycles in lake sediments.

Keywords: Dissolved organic matter; goethite; adsorption; parallel factor analysis; Potamogeton malaianus; Microcystis aeruginosa

近年来全球淡水湖泊富营养化加剧, 浮游藻类和水生植物过度生长, 引起广泛关注 ${ }^{[1]}$. 藻类和植物的生 长、衰亡、裂解等过程会释放大量的自生源可溶有机质 (DOM) 进人湖泊水体, 造成背景浓度显著升高 ${ }^{[2]}$. DOM 的结构复杂, 富含蛋白质、腐殖质和多糖等亲水、疏水物质, 这些组分内部的化学基团赋予 DOM 很强的 生物、化学活性 ${ }^{[3]}$. 其中, 化学吸附是影响 DOM 浓度、组成及结构的重要环境行为之一 ${ }^{[4]}$.

DOM 在沉积物界面的吸附行为不仅是影响湖泊碳库的关键因素, 而且也可改变沉积物的有机组成, 从 而影响无机、有机污染物的生物地球化学循环 ${ }^{[5-6]}$. 针铁矿是沉积物中普遍存在的一种较稳定的铁氧化矿 物, 由进人湖区沉积物上层的铁质溶解, 在氧化条件下形成三价态, 并与氧、氢氧根离子结合形成, 在太湖、 滇池等富营养化湖泊中分布广泛 ${ }^{[7-8]}$. 同时, 因其比表面积较高, 反应活性较强, 针铁矿对 DOM 及有机污染 物等具有很强的吸附潜力 ${ }^{[6]}$. DOM 中不同分子对矿物的亲和力不同, 因此与矿物接触时亲和力较高的分子 通常优先被吸附, 而亲和力较弱的分子仍留在水相中, 从而造成非均质吸附. 据文献报道 ${ }^{[9-10]}$, 富含芳香族、 羧基以及疏水物质的 DOM 对铁铝氧化物的亲和力很强; 也有研究表明针铁矿对胡敏酸中大分子组分的吸 附能力较强 ${ }^{[11]}$, 而 DOM 中氧基含量低 (醇和醚) 的小分子组分不易被氢氧化铁吸附 ${ }^{[10]}$. 然而, 目前针铁矿 对自生源 DOM 的吸附研究很少. 与腐殖质相比, 藻源 DOM (ADOM) 和草源 DOM (MDOM) 相对新鲜, 富含类 蛋白物质, 腐殖度很低, 因此它们与针铁矿的相互作用可能不同于以往的腐殖质研究. 特别是, 随着蓝藻水 华暴发和水生植物过度生长加剧, ADOM 和 MDOM 已成为湖泊碳库的重要来源, 有必要深人研究它们在泥 水界面的吸附行为.

三维苂光光谱法 (EEM) 是一种快速、灵敏、无损地分析 DOM 组成和结构变化的常用方法 ${ }^{[12-14]}$. 不过由 于 DOM 的 EEM 图谱复杂, 不同苂光物质的苂光峰可能重叠, 导致后续分析困难. 近年来, EEM 结合平行因 子分析 (EEM-PARAFAC) 可从多个 EEM 数据集中提取最小残差的独立苂光组分, 有利于跟踪分析不同 DOM 组分的环境行为 ${ }^{[3,15]}$, 也为研究 DOM 组分在针铁矿表面的吸附分离特征提供了契机. 本研究选取太湖 藻型湖区的铜绿微囊藻 (Microcystis aeruginosa) 和草型湖区的马来眼子菜 (Potamogeton malaianus) 作为代表, 通过批量实验考察针铁矿对 ADOM 和 MDOM 的吸附行为, 运用吸附动力学和吸附等温线模型分析 DOM 的 吸附过程, 并结合 EEM-PARAFAC 解析不同 DOM 组分的非均质吸附特征, 为理解富营养化湖泊中自生源 DOM 与沉积物矿物的相互作用及对污染物迁移转化的影响特征提供理论依据.

\section{1 材料和方法}

\section{1 针铁矿和 DOM 的制备}

以 $\mathrm{Fe}\left(\mathrm{NO}_{3}\right)_{3}$ 和 $\mathrm{NaOH}$ 为原料, 按碱性法制备针铁矿 ${ }^{[16]}$. 该方法制备的针铁矿颗粒尺寸大于天然针铁 矿 $^{[17]}$. 经 X 射线衍射和红外光谱鉴定, 合成产物主要为 $\alpha-\mathrm{FeOOH}$. 经 $\mathrm{N}_{2}$ 吸附 BET 法测定针铁矿的表面积为 $45.08 \mathrm{~m}^{2} / \mathrm{g}$ ( ASAP 2020, Micromeritics, USA).

于 2017 年 8 月在太湖梅梁湾和胥口湾分别采集铜绿微囊藻浆和马来眼子菜作为浮游藻类和水生植物

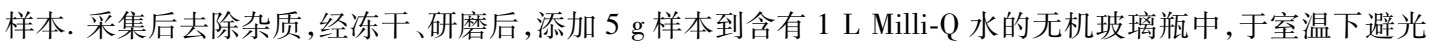
振荡 ${ }^{[14]}$. 经 5 天后, DOM 释放量达到峰值时, 离心、过滤 $(0.7 \mu \mathrm{m}$ 玻璃纤维滤膜, 预烧) 上清液, 将滤液置于 $4^{\circ} \mathrm{C}$ 条件下透析以去除无机盐和小分子有机物. 透析袋孔径为 $3500 \mathrm{D}^{[18]}$, 透析时间为 $24 \mathrm{~h}$, 重复 3 次. 经测 定, 透析过程中 ADOM 和 MDOM 的 DOC 浓度损失分别为 $15 \%$ 和 $11 \%$. 最终, 将得到的 2 种 DOM 溶液冻存 备用.

\section{2 吸附动力学和等温线}

分别取 $30 \mathrm{~mL}$ 的 ADOM 和 MDOM 溶液 (DOC 浓度为 $30 \mathrm{mg} / \mathrm{L}$ ) 置于一系列 $50 \mathrm{~mL}$ 无机玻璃管中, 同时 添加 $1.30 \mathrm{~g} / \mathrm{L}$ 针铁矿、 $0.01 \mathrm{~mol} / \mathrm{L} \mathrm{NaCl}$ 及 $100 \mathrm{mg} / \mathrm{L} \mathrm{NaN}_{3}$ (微生物抑制剂), 用 $\mathrm{HCl}$ 和 $\mathrm{NaOH}$ 调节 $\mathrm{pH}$ 值为 7.0 0.2 . 用聚四氟乙烯塞封口后, 在 $25^{\circ} \mathrm{C}$ 条件下避光振荡 $(120 \mathrm{r} / \mathrm{min}$ ). 分别于第 $0.25 、 0.5 、 1 、 2 、 4 、 6 、 12 、 24 \mathrm{~h}$ 
取出样品, 离心、过滤 $(0.45 \mu \mathrm{m}$ 玻璃纤维滤膜) 上清液, 测定滤液的 DOC 浓度.

在相同实验条件下, 以针铁矿浓度为 $1.30 \mathrm{~g} / \mathrm{L}, \mathrm{DOC}$ 浓度为 $0 \sim 70 \mathrm{mg} / \mathrm{L}$, 开展针铁矿对 ADOM 和 MDOM 的吸附等温线研究. 依据动力学实验结果设定平衡时间为 $8 \mathrm{~h}$, 待吸附平衡后取出样品, 离心、过滤上清液, 测定最终滤液的 DOC 浓度和 EEM 光谱. 以 DOC 浓度为零作为对照组. 另外, 收集离心管中残留的针铁矿样 品, 测定红外光谱. 本研究中实验均设置 3 个平行样, 取平均值.

\section{3 分析方法}

DOC 浓度通过总有机碳分析仪检测不可吹脱有机碳 (TOC-Vcph, 岛津) 测得. DOM 吸收光谱由 UV-Vis 光谱仪 (UV-2550, 岛津) 测定, 检测单元长度 $1 \mathrm{~cm}$, 波长范围 200 $800 \mathrm{~nm}$, 间隔 $1 \mathrm{~nm}$, 狭缝宽度 $1 \mathrm{~nm}$, 扫描速 度 $210 \mathrm{~nm} / \mathrm{min}$. 根据 $254 \mathrm{~nm}$ 处的吸收值和 DOC 浓度计算 $\mathrm{SUVA}_{254}{ }^{[19]}$. 运用指数函数对 $275 \sim 295$ 和 350 $400 \mathrm{~nm}$ 的吸收光谱进行非线性拟合得到 $S_{275-295}$ 和 $S_{350-400}$, 计算两者的比值为 $S_{\mathrm{R}}{ }^{[19]}$. EEM 光谱通过荧光光谱 仪 (F-7000, 日立) 测定, 灯源为 $700 \mathrm{~V}$ 氙灯, 扫描发射波长 $250 \sim 550 \mathrm{~nm}$, 间隔 $1 \mathrm{~nm}$, 扫描激发波长 $200 \sim 450$ $\mathrm{nm}$, 间隔 $5 \mathrm{~nm}$, 扫描速度 $2400 \mathrm{~nm} / \mathrm{min}$, 激发和发射狭缝带宽均为 $5 \mathrm{~nm}$, 以 Milli-Q 水作为空白参比. 采用 MATLAB 的 drEEM 工具箱对 EEM 光谱依次进行水拉曼散射峰校正、内滤效应校正及瑞利散射效应校正, 并 将校正后的荧光强度标准化为 Raman Unit $275 \mathrm{~nm}\left(\mathrm{RU}_{275}\right)$ 消除灯源的日常误差 ${ }^{[20]}$. 通过计算激发波长为 $254 \mathrm{~nm}$, 发射扫描区域分别为 $435 \sim 480$ 和 $300 \sim 345 \mathrm{~nm}$ 的苂光强度比值获得腐殖度指数 $(H I X)^{[19]}$. 将吸附 前后的针铁矿样品冻干、混匀, 通过 Smart iTR ATR Nicolet iS 10 FTIR 光谱仪 (Thermo Nicolet, USA) 采集红 外光谱, 扫描波长 $4000 \sim 650 \mathrm{~cm}^{-1}$, 扫描次数 64 次, 分辨率 $4 \mathrm{~cm}^{-1}$. 使用 Nicolet OMNIC 软件 (Thermo, USA) 对采集的红外光谱进行平滑处理和基线校正,并转换成吸光度.

\section{4 数值分析}

1.4.1 EEM-PARAFAC 分析 PARAFAC 分析是通过交替最小二乘法把所有样品的 EEM 数据矩阵分解成 3 个线性项和 1 个残留数组,进而分离出数学和化学独立的组分 (每个组分代表单独或强烈共变化的苂光 团 ${ }^{[20]}$. 矩阵公式为:

$$
X_{i j k}=\sum_{f=1}^{F} a_{i f} \cdot b_{i f} \cdot c_{k f}+e_{i j k}
$$

式中, $i=1 、 2 、 \cdots 、 I ; j=1 、 2 、 \cdots 、 J ; k=1 、 2 、 \cdots 、 K ; f$ 为某个苂光组分; $F$ 为苂光组分总数目; $e$ 为残差. 用 drEEM 工具箱对 EEM 进行运算,并用残差分布和 “S4T6C3” 算法验证模型有效性. 设定每个苂光组分的最 大苂光强度 $\left(F_{\text {max }}\right)$ 与其浓度呈固定比例.

1.4.2 吸附动力学模型 采用伪一级动力学模型拟合吸附过程, 该模型已广泛用于考察无机矿物固相界面的 吸附行为 ${ }^{[21]}$, 公式为:

$$
Q_{\mathrm{t}}=Q_{\mathrm{e}}\left(1-\mathrm{e}^{-k_{i} t}\right)
$$

式中, $Q_{t}$ 为 $t(\mathrm{~h})$ 时针铁矿对 DOM 的吸附量 $(\mathrm{mg} / \mathrm{g}), Q_{\mathrm{e}}$ 为平衡时针铁矿对 DOM 的吸附量 $(\mathrm{mg} / \mathrm{g}), k_{\mathrm{t}}$ 为伪一 级动力学常数 $\left(\mathrm{h}^{-1}\right)$.

1.4.3 吸附等温线模型 分别采用 Langmuir 和 Freundlich 模型拟合吸附等温线, 其中前者适于描述匀质表面 的吸附数据, 而后者适于表征非匀质表面的吸附行为 ${ }^{[22-23]}$. Langmuir 和 Freundlich 模型如式 (3) 和式 (4) 所示:

$$
\begin{gathered}
Q_{\mathrm{e}}=\frac{Q_{\text {max }} \cdot K_{\mathrm{L}} \cdot C_{\mathrm{e}}}{1+K_{\mathrm{L}} \cdot C_{\mathrm{e}}} \\
Q_{\mathrm{e}}=K_{\mathrm{f}} \cdot C_{\mathrm{e}}^{N}
\end{gathered}
$$

式中, $Q_{\mathrm{e}}$ 为平衡浓度为 $C_{\mathrm{e}}(\mathrm{mg} / \mathrm{L})$ 时针铁矿对 DOM 的吸附量 $(\mathrm{mg} / \mathrm{g}), Q_{\text {max }}$ 为饱和吸附量 $(\mathrm{mg} / \mathrm{g}), K_{\mathrm{L}}$ 为与吸 附力有关的 Langmuir 吸附平衡常数 $(\mathrm{L} / \mathrm{mg}), K_{\mathrm{f}}$ 为 Freundlich 吸附平衡常数 $\left((\mathrm{mg} / \mathrm{g}) /(\mathrm{mg} / \mathrm{L})^{N}\right), N$ 为非线 性系数.

\section{2 结果与讨论}

\subsection{DOM 表征}

2 种 DOM 的 UV-Vis 光谱如图 1a 所示, 随波长增加, DOM 的吸光度逐渐降低. MDOM 的吸光度高于 
$\mathrm{ADOM}$, 表明含有更多的芳香物质和官能团. 与此一致, ADOM 的 $\mathrm{SUVA}_{254}$ 显著低于 MDOM (表 1). 通常 $\mathrm{SUVA}_{254}<3 \mathrm{~L} /(\mathrm{mg} \cdot \mathrm{m})$ 说明 $254 \mathrm{~nm}$ 处主要为无吸收的脂肪族物质, 但也可能存在一定的芳香性结构, 而 $>3 \mathrm{~L} /(\mathrm{mg} \cdot \mathrm{m})$ 则意味着具有相当含量的芳香疏水物质 ${ }^{[19]} . S_{\mathrm{R}}$ 值一般与 DOM 平均分子量呈反比, 故 ADOM 的平均分子量较高, 这可能与藻体释放的生物大分子有关. 如图 1b 和 1c 所示, 2 种 DOM 的 EEM 图谱中主 要苂光峰的发射波长均小于 $380 \mathrm{~nm}$, 与羟基、氨基等官能团相关. 据文献报道 ${ }^{[3]}$, 类腐殖苂光团的发射波长 往往大于 $380 \mathrm{~nm}$, 而小于 $380 \mathrm{~nm}$ 的菼光团主要为类蛋白物质. MDOM 的 HIX 值高于 ADOM, 这与前人研究 发现藻型湖区沉积物 DOM 中类蛋白物质含量高于草型湖区沉积物 DOM 的报道一致 ${ }^{[24]}$.
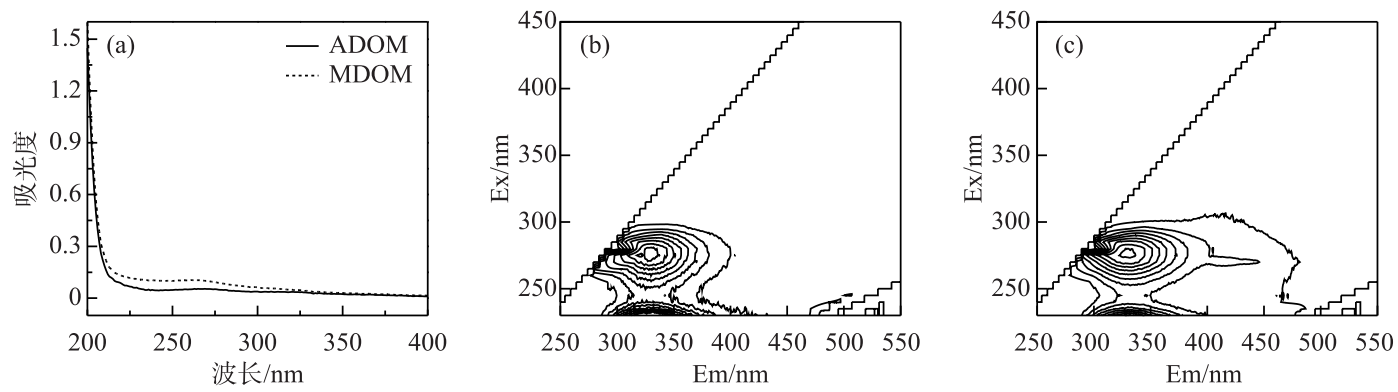

图 1 ADOM 和 MDOM 的光谱图: (a)UV-Vis 光谱, (b) 和 (c) EEM 光谱

Fig. 1 Spectra of ADOM and MDOM: (a) UV-Vis spectra, (b) and (c) EEM spectra

表 1 ADOM 和 MDOM 的光谱特征

Tab.1 Optical characteristics of ADOM and MDOM

\begin{tabular}{ccccccccc}
\hline \multirow{2}{*}{ DOM 类型 } & \multirow{2}{*}{$\mathrm{SUVA}_{254} /(\mathrm{L} /(\mathrm{mg} \cdot \mathrm{m}))$} & $S_{\mathrm{R}}$ & & & \multicolumn{5}{c}{ 各组分占比/\% } \\
\cline { 5 - 8 } & & & & $\mathrm{C} 1$ & $\mathrm{C} 2$ & $\mathrm{C} 3$ & $\mathrm{C} 4$ \\
\hline \multirow{2}{*}{$\mathrm{ADOM}$} & 0.75 & 0.99 & 0.13 & 5.0 & 24.0 & 69.0 & 2.0 \\
$\mathrm{MDOM}$ & 1.58 & 1.18 & 0.39 & 20.6 & 12.4 & 57.4 & 9.6 \\
\hline
\end{tabular}

运用 EEM-PARAFAC 分析共得到 4 个苂光组分, 经半检验分析证实该模型有效 (图 2). 参考前人研究 基于苂光峰的位置识别苂光组分 ${ }^{[15,25]}$, 组分 C1 在 Ex $240,260 / \mathrm{Em} 440 \mathrm{~nm}$ 处存在两个苂光峰, 可归为类富 里酸组分. 组分 C2 分别在 Ex 230, 265/Em $302 \mathrm{~nm}$ 处中存在菼光峰, 与类蛋白物质中的类酪氨酸组分相似. 组分 C3 的两个苂光峰分别位于 Ex $230,275 / \mathrm{Em} 332 \mathrm{~nm}$, 可归为类色氨酸物质. 组分 C4 的菼光峰位于 Ex $280,360 / \mathrm{Em} 448 \mathrm{~nm}$, 与类胡敏酸物质的菼光特征相似. 这 4 个菼光组分均已在太湖水体中检出 ${ }^{[2]}$, 说明浮 游藻类和水生植物是 DOM 的重要来源. 根据各个组分的 $F_{\text {max }}$ 值, 发现类蛋白组分 ( C2 和 C3 ) 是 ADOM 和 MDOM 的主要荧光物质, 分别占总苂光组分的 $93 \%$ 和 70\%, 而类腐殖质物质, 尤其是长发射波长的类腐殖质 组分的含量很低. 与陆源输人为主的河流、污水及土壤 DOM 等相比 ${ }^{[25-27]}$, 藻源和草源 DOM 相对新鲜, 类蛋 白含量高, 腐殖度低. 这些特有的组分特征可能导致它们与针铁矿的吸附作用区别于典型陆源 DOM 的 研究.

\section{$2.2 \mathrm{DOM}$ 吸附动力学}

如图 3a 所示, 随时间推移, 针铁矿对 2 种 DOM 的吸附量先快速升高, 而后逐渐平衡, 表观吸附平衡时间 为 $8 \mathrm{~h}$, 与前人报道相近 ${ }^{[11]}$. 初期的快吸附阶段主要为表面吸附, 受 DOM 浓度和针铁矿固相活性位点的双 重影响, 而慢平衡阶段则因有效吸附位点被逐渐占据而趋于平衡. 采用伪一级动力学方程较好地拟合了针 铁矿对 ADOM 和 MDOM 的吸附过程 $\left(R^{2}=0.96\right)$ (表 2). ADOM 的 $K_{\mathrm{t}}$ 值和 $Q_{\mathrm{e}}$ 值均高于 MDOM, 表明针铁矿 不仅对 ADOM 的吸附速率快, 且平衡吸附量也较高. 虽然 ADOM 和 MDOM 都是自生源 DOM, 但其组分结构 不同,故对针铁矿的吸附亲和力也不同. 

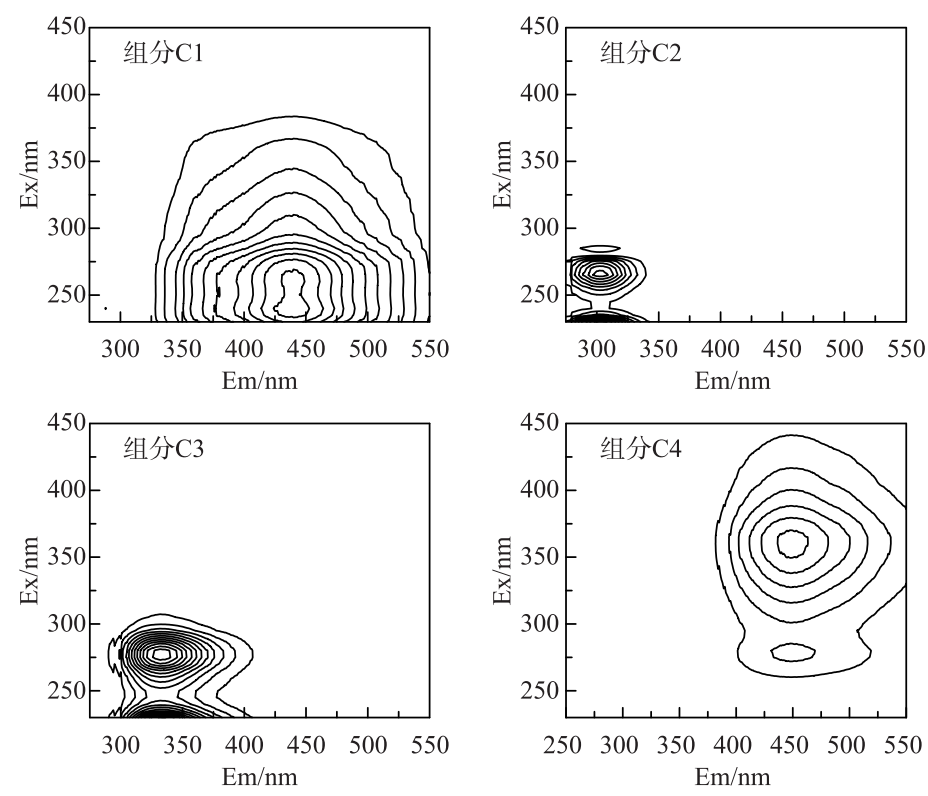

图 2 PARAFAC 组分的 EEM 光谱

Fig.2 EEM spectra of PARAFAC components

表 2 针铁矿对 ADOM 和 MDOM 的吸附动力学和吸附等温线拟合结果

Tab.2 Fitting results of adsorption kinetics and isotherms of ADOM and MDOM on goethite

\begin{tabular}{|c|c|c|c|c|c|c|c|c|c|}
\hline \multirow{2}{*}{$\begin{array}{l}\text { DOM } \\
\text { 类型 }\end{array}$} & \multicolumn{3}{|c|}{ 伪一级动力学方程 } & \multicolumn{3}{|c|}{ Langmuir 吸附等温线 } & \multicolumn{3}{|c|}{ Freundlich 吸附等温线 } \\
\hline & $\begin{array}{c}Q_{\mathrm{e}} / \\
(\mathrm{mg} / \mathrm{g})\end{array}$ & $\begin{array}{l}k_{t} / \\
\mathrm{h}^{-1}\end{array}$ & $R^{2}$ & $\begin{array}{c}Q_{\max } / \\
(\mathrm{mg} / \mathrm{g})\end{array}$ & $\begin{array}{c}K_{\mathrm{L}} / \\
(\mathrm{L} / \mathrm{mg})\end{array}$ & $R^{2}$ & $\begin{array}{c}K_{\mathrm{f}} / \\
\left((\mathrm{mg} / \mathrm{g}) /(\mathrm{mg} / \mathrm{L})^{N}\right)\end{array}$ & $N$ & $R^{2}$ \\
\hline ADOM & 11.85 & 2.32 & 0.96 & 23.77 & 0.15 & 0.94 & 4.28 & 0.44 & 0.91 \\
\hline MDOM & 9.18 & 2.23 & 0.96 & 19.10 & 0.18 & 0.92 & 4.00 & 0.40 & 0.92 \\
\hline
\end{tabular}

\section{$2.3 \mathrm{DOM}$ 吸附等温线}

不同初始浓度下 2 种 DOM 的平衡吸附量如图 3b 所示. 随着初始浓度增加, 平衡吸附量先剧烈升高, 然 后逐渐趋于饱和. 该过程说明 DOM 极易被针铁矿吸附, 但吸附能力有限. Langmuir 等温线模型拟合性较好 $\left(R^{2} \geqslant 0.92\right)$, 其中 $\mathrm{ADOM}$ 的 $Q_{\text {max }}$ 较高, $K_{\mathrm{L}}$ 值较低, 表明其对针铁矿的亲和力高于 $\mathrm{MDOM}$, 这与吸附动力学结 果一致. 同时, Freundlich 等温线模型也较好地拟合了吸附过程 $\left(R^{2} \geqslant 0.91\right)$. 与 Langmuir 相比, Freundlich 模 型更适于描述非匀质表面的吸附数据, 且 $N<1$, 这是由于针铁矿表面的吸附位点不均匀以及 DOM 的组成 复杂, 导致吸附非线性很强. 通常 DOM 在介质上的吸附过程均呈非线性, 且分子量大、疏水性强的 DOM 含 有更多的共轭双键, 疏水作用和范德华力的吸引点位增多, 从而更易被吸附 ${ }^{[22]}$. 本研究中, 针铁矿对平均分 子量较高、芳香度较低的 ADOM 的吸附量高于 MDOM, 这虽与前人研究发现胡敏酸中高摩尔质量、低芳香度 的组分优先被针铁矿吸附的报道一致 ${ }^{[11]}$, 但 ADOM 中类胡敏酸和类富里酸组分含量远低于 MDOM, 说明类 腐殖组分并非吸附量的决定性因素, 可能更应归因于 DOM 中其他组分的差异. 不同 DOM 组分的固有组成 和结构使其在介质表面的吸附机制不同. 据报道,蛋白质和核酸分子主要依靠电子作用力吸附到针铁矿表 面 $^{[28]}$, 而蛋白质分子末端的磷酸基也可通过配位基交换与铁矿物表面形成磷铁键 ${ }^{[17]}$. 此外, 含硫的氨基酸 (如半胱氨酸) 也能与铁氧化物发生强烈相互作用 ${ }^{[29]}$. 因此, 2 种 DOM 中较为丰富的类蛋白组分可能是造成 针铁矿对其吸附量差异的主要原因.

\subsection{PARAFAC 组分的吸附}

进一步分析吸附过程中 DOM 结构及 PARAFAC 组分的变化. 经针铁矿吸附后, 2 种 DOM 中类蛋白组分 

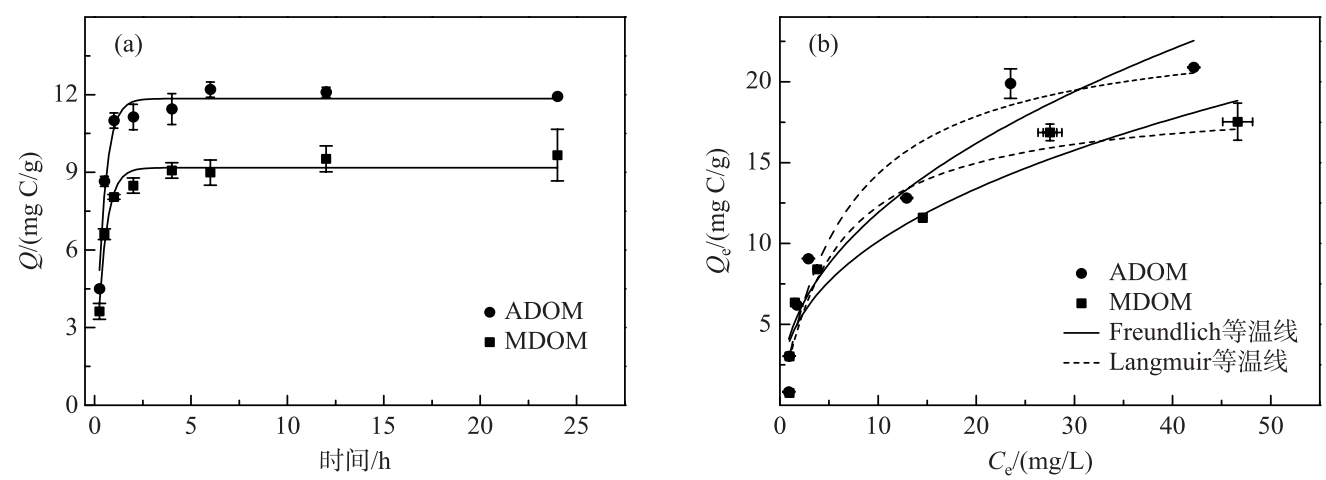

图 3 针铁矿对 ADOM 和 MDOM 的吸附动力学 (a) 和吸附等温线 (b)

Fig.3 Adsorption kinetics (a) and adsorption isotherms (b) of ADOM and MDOM on goethite

C2、C3 的相比比例显著下降, 而类腐殖酸组分 C1、C4 的相对比例明显升高 (图 4a). DOM 浓度和类蛋白组分 的同步降低表明针铁矿吸附了 DOM 中大量的类色氨酸和类酪氨酸组分, 而对类富里酸和类胡敏酸组分的 吸附量有限, 从而 $\mathrm{C} 1 、 \mathrm{C} 4$ 的相对比例显著升高. 与此一致, 残留 DOM 的 $\mathrm{SUVA}_{254}$ 和 $S_{\mathrm{R}}$ 均显著高于原始 DOM, 证实芳香度较高、平均分子量较低的 DOM 组分保留在液相中 (图 4b). 进一步拟合 PARAFAC 组分的吸附等 温线, 发现 2 种 DOM 中 $\mathrm{C} 2 、 \mathrm{C} 3$ 的 $K_{\mathrm{f}}$ 值均显著高于 $\mathrm{C} 1$ 和 $\mathrm{C} 4$, 证实了针铁矿对类蛋白组分的吸附量高于类腐 殖组分 (表 3). 不过, 由于 ADOM 和 MDOM 中类腐殖组分的初始含量远低于类蛋白组分, 故两种组分的吸 附量差异并非完全由吸附亲和力决定, 也与初始浓度密切有关. 根据吸附等温线, 在一定浓度范围内, 吸附 质的吸附量与其初始浓度呈正比. 前人研究大多采用标准腐殖质或以腐殖质为主要成分的 DOM, 吸附过程 由腐殖质主导 ${ }^{[9,11]}$. 然而, 在以蛋白质、多糖和脂类为主要成分的微生物胞外聚合物研究中发现蛋白质在针 铁矿吸附过程中占主要作用, 特别是含有芳香性氨基酸的蛋白质分子更易被吸附 ${ }^{[17]}$. 本研究中自生源 DOM 的主要苂光组成 $(\mathrm{C} 2 、 \mathrm{C} 3$ ) 均为类芳香族氨基酸组分 (类酪氨酸和类色氨酸), 所以呈现出较强的吸附特征. 此外, 与类蛋白物质相比, $\mathrm{C} 1 、 \mathrm{C} 4$ 的 $N$ 值更接近于 1 , 说明类腐殖物质在针铁矿上的吸附近似线性. 这可能是 由于类蛋白物质受吸附点位限制效应影响较大, 在负荷较低时更易被针铁矿吸附, 所以吸附呈非线性 ${ }^{[30]}$. 被吸附 DOM 中类蛋白组分和类腐殖组分的比值 $(\mathrm{C} 2+\mathrm{C} 3) /(\mathrm{C} 1+\mathrm{C} 4)$ 变化也证实了这一趋势 ${ }^{[15]}$. 如图 4c 所 示, 随吸附量升高, 被吸附 DOM 部分的 $(\mathrm{C} 2+\mathrm{C} 3) /(\mathrm{C} 1+\mathrm{C} 4)$ 显著降低. 这表明吸附初期针铁矿表面有效位点 较多, 类蛋白物质几乎完全被吸附到针铁矿表面, 而随着针铁矿表面被越来越多的 DOM 分子覆盖, 受吸附 点位 (如配位基团) 限制效应影响,类蛋白物质的吸附程度逐渐下降.

(a)

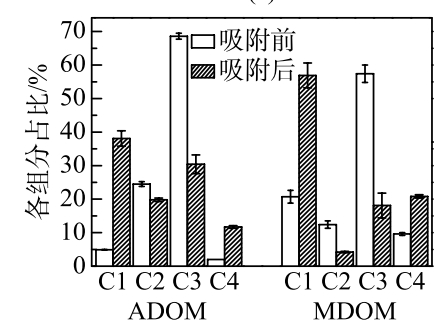

(b)

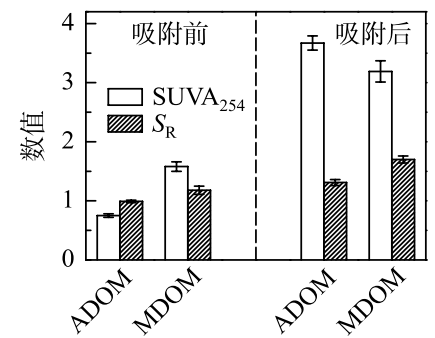

(c)

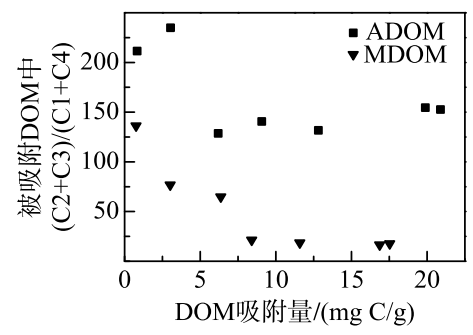

图 4 吸附前后 ADOM 和 MDOM 中 4 个 PARAFAC 组分的相对比例(a), 吸附前后 $\mathrm{ADOM}$ 和 MDOM 的 $\mathrm{SUVA}_{254}$ 和 $S_{\mathrm{R}}$ 变化 (b),

以及被吸附 DOM 中蛋白类组分与腐殖类组分的比值随 DOM 吸附量的变化 $(\mathrm{c})$

Fig.4 Proportions of four PARAFAC components in ADOM and MDOM before and after adsorption(a),

$\mathrm{SUVA}_{254}$ and $S_{\mathrm{R}}$ values of ADOM and MDOM before and after adsorption (b), and $(\mathrm{C} 2+\mathrm{C} 3) /(\mathrm{C} 1+\mathrm{C} 4)$ ratios of adsorbed DOM with increasing adsorption $\operatorname{amount}(\mathrm{c})$ 
表 3 针铁矿吸附 4 个 PARAFAC 组分的 Freundlich 等温线参数

Tab.3 Freundlich isotherm parameters of four PARAFAC components adsorbed on goethite

\begin{tabular}{|c|c|c|c|c|c|c|c|c|}
\hline \multirow{2}{*}{ 参数 } & \multicolumn{4}{|c|}{ ADOM } & \multicolumn{4}{|c|}{ MDOM } \\
\hline & C1 & $\mathrm{C} 2$ & C3 & $\mathrm{C} 4$ & $\mathrm{C} 1$ & $\mathrm{C} 2$ & $\mathrm{C} 3$ & $\mathrm{C} 4$ \\
\hline$K_{\mathrm{f}} /\left((\mathrm{mg} / \mathrm{g}) /(\mathrm{mg} / \mathrm{L})^{N}\right)$ & 0.016 & 3.448 & 7.195 & 0.246 & 0.006 & 3.386 & 3.765 & 0.393 \\
\hline$N$ & 1.12 & 0.71 & 0.69 & 0.99 & 1.31 & 0.84 & 0.59 & 1.31 \\
\hline$R^{2}$ & 0.802 & 0.950 & 0.967 & 0.987 & 0.825 & 0.928 & 0.974 & 0.889 \\
\hline
\end{tabular}

$\mathrm{C} 3$ 的 $K_{\mathrm{f}}$ 值高于 $\mathrm{C} 2$, 而 $\mathrm{C} 2$ 的 $N$ 值比 $\mathrm{C} 3$ 更接近于 1 , 意味着类色氨酸物质对针铁矿的亲和力高于类酪氨 酸物质, 且吸附非线性更强. 通常, 荧光光谱红移表明 DOM 组分中具有更多的饱和凝聚结构, 分子质量更 高. C3 的发射波长长于 $\mathrm{C} 2$, 即类色氨酸组分具有较大的分子量和较高的结构缩合度. 前人采用尺寸排阻色 谱指出蛋白质中的高摩尔质量分子和磷酸大分子优先被针铁矿吸附 ${ }^{[28]}$, 而富有胺基和羧化物的复杂氨基酸 对针铁矿的亲和力也通常高于结构简单的氨基酸 ${ }^{[31]}$. 此外, 针铁矿对蛋白质中的芳香氨基酸组分吸附能力 强于非芳香组分 ${ }^{[17]}$, 而 $\mathrm{C} 3$ 的芳香度较强,因此吸附量更高.

尽管 2 种 DOM 中 $\mathrm{C} 4$ 的相对含量低于 $\mathrm{C} 1$, 但其 $K_{\mathrm{f}}$ 值仍较高, 说明与类富里酸组分相比, 类胡敏酸组分 更易被针铁矿吸附. 这种非均质吸附特征与 2 种组分的分子量、基团差异及疏水性有关. 胡敏酸结构密实, 平均分子质量较大, 含有更多与针铁矿具有亲和力的反应基团 (如羧基等) ${ }^{[9]}$. 相较而言, 富里酸分子尺寸较 小, 酸度较高 ${ }^{[32]}$. 此外, C4 的疏水性强于 $\mathrm{C} 1$, 更有利于通过疏水作用与针铁矿相互作用. 不过, ADOM 和 MDOM 中大量类蛋白物质的竞争吸附也可能影响针铁矿对类富里酸和类胡敏酸组分的吸附亲和力 ${ }^{[33]}$. 总 体而言, 针铁矿对 DOM 的非均质吸附与组分含量、分子质量、芳香度、及表面有效位点密切相关. 同时, 这种 非均质吸附也意味着虽然 DOM 是一种由类蛋白物质和类腐殖质物质通过弱离散力聚集的超分子组合 体 ${ }^{[33]}$,但一旦与针铁矿接触,这种超分子组合体极易解体,从而不同组分产生非线性吸附.

\section{5 红外光谱}

针铁矿吸附 2 种 DOM 前后的红外光谱如图 5 所 示. 原始针铁矿在 1230 和 $3094 \mathrm{~cm}^{-1}$ 波长处存在表 面一 $\mathrm{OH}$ 的弯曲振动和伸缩振动特征峰, $1373 \mathrm{~cm}^{-1}$ 处 存在针铁矿的特征峰 $(\mathrm{Fe}-\mathrm{OH} \text { 弯曲模态 })^{[34]}$. 而针铁矿 吸附 DOM 后,其红光光谱发生明显变化,证明针铁矿 吸附 DOM 属于化学作用. 位于 $3094 \mathrm{~cm}^{-1}$ 的吸收峰强 度降低,说明针铁矿表面的一 $\mathrm{OH}$ 参与吸附过程. 1770 $\mathrm{cm}^{-1}$ 处的一 $\mathrm{COOH}$ 特征峰强度升高, 表明羧基是吸附 过程中重要官能团. 有文献指出 DOM 中羧基和羟基之 间的配位交换是矿物吸附的机制之一 ${ }^{[35]} .1550 \mathrm{~cm}^{-1}$ 处 的氨基 II 的 $\mathrm{N}-\mathrm{H}$ 弯曲和 $\mathrm{C}-\mathrm{N}$ 伸缩振动特征峰的强 度均升高,证实吸附 DOM 后针铁矿表面被蛋白类物质 覆盖. $1373 \mathrm{~cm}^{-1}$ 处的特征峰消失, 意味着 DOM 中的活

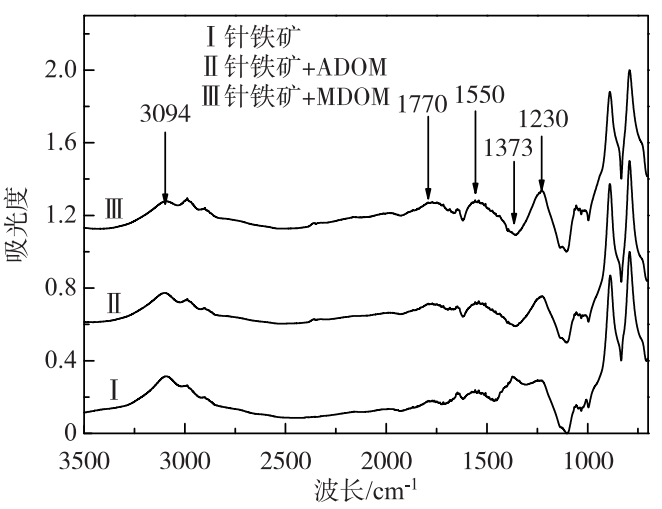

图 5 针铁矿吸附 DOM 前后的红外光谱

Fig. 5 Infrared spectra of goethite before and after DOM adsorption 性基团与针铁矿发生配位交换作用. 以上结果表明氨 基、羧基与表面活性基团 (如羟基) 的配位交换及络合是 ADOM 和 MDOM 与针铁矿结合的重要机制.

针铁矿吸附 DOM 后其表面有机组分发生非均质改变, 影响其生物化学活性. 针铁矿优先吸附 ADOM 和 MDOM 中的类蛋白物质,这可能是前人研究发现太湖沉积物 DOM 富含蛋白类组分的重要原因之一 ${ }^{[26]}$. 通 常, 类蛋白物质的生物活性高于类腐殖物质 ${ }^{[27]}$, 因此针铁矿吸附的大量蛋白类组分可为其表面附着的微生 物提供碳源和能量,进而改变群落结构和功能. 此外, DOM 也可能影响针铁矿对重金属及有机污染物的吸 附作用. 据文献报道 ${ }^{[36]}$, 基于类蛋白物质对新型有机污染物 (特别是抗生素) 具有很强的络合作用,针铁矿 吸附 DOM 后其表面的有效吸附位点增加,从而污染物的钝化作用增强. 但是, 因存在无机离子的干扰可能 
性, 分子光谱 (尤其是吸收和荧光光谱) 仅能够提供针铁矿与 DOM 相互作用的宏观信息,具有一定的不确定 性. 未来有必要采用更准确的手段(如高分辨率质谱和同位素等) 对 ADOM 和 MDOM 的吸附特征进一步探 究. 总体而言, 蓝藻水华暴发和水生植物过量生长产生的自生源 DOM 可以覆盖沉积物矿物表面, 对湖泊沉 积物的生物地球化学行为具有重要影响.

\section{3 结论}

1) ADOM 和 MDOM 类蛋白组分含量均高于类腐殖组分, 且 ADOM 中类蛋白质组分含量更高.

2) 针铁矿对 2 种 DOM 的吸附过程符合伪一级动力学, 其中类蛋白物质含量更高的 ADOM 吸附速率更 快; Langmuir 和 Freundlich 吸附等温线可表征吸附热力学, 且 ADOM 的饱和吸附量高于 MDOM.

3) DOM 中不同组分的吸附量与组分浓度和结构有关, 类蛋白物质是针铁矿吸附 ADOM 和 MDOM 过程 中的优势组分,其中分子量较大、芳香度较高的类色氨酸组分的吸附量高于类酪氨酸组分.

4 ) DOM 中氨基、羧基等官能团是与针铁矿发生界面吸附反应的重要活性基团.

\section{4 参考文献}

[ 1 ] Wang YP, Zhu GW, Hong DL et al. Environmental characteristics of sediment-water interface of phytoplankton and macrophyte dominated zones in Lake Taihu. J Lake Sci, 2013, 25(2) : 199-208. DOI: 10.18307/2013.0204. [王永平, 朱广 伟, 洪大林等. 太湖草/藻型湖区沉积物-水界面环境特征差异. 湖泊科学, 2013, 25(2) : 199-208. ]

[ 2 ] Zhou YQ, Jeppesen E, Zhang YL et al. Chromophoric dissolved organic matter of black waters in a highly eutrophic Chinese lake: Freshly produced from algal scums? Journal of Hazardous Materials, 2015, 299: 222-230.

[ 3 ] Ishii SKL, Boyer TH. Behavior of reoccurring PARAFAC components in fluorescent dissolved organic matter in natural and engineered systems: A critical review. Environmental Science \& Technology, 2012, 46(4) : 2006-2017.

[ 4 ] Avneri-Katz S, Young RB, Mckenna AM et al. Adsorptive fractionation of dissolved organic matter (DOM) by mineral soil: Macroscale approach and molecular insight. Organic Geochemistry, 2017, 103: 113-124.

[ 5 ] Liang P, Li YC, Zhang C et al. Effects of salinity and humic acid on the sorption of $\mathrm{Hg}$ on Fe and Mn hydroxides. Journal of Hazardous Materials, 2013, 244: 322-328.

[ 6 ] Guo XT, Zhang J, Ge JH et al. Sorption and photodegradation of tylosin and sulfamethazine by humic acid-coated goethite. Rsc Adv, 2015, 122(5): 100464-100471.

[ 7 ] Hung ZC, Liu GB. Characteristics of ferruginous concretions in modern sediments of the Taihu Lake: Implications for the origin of Lake Taihu. Acta Sedimentologica Sinica, 2014, 32(3) : 503-509. [黄志诚, 刘冠邦. 太湖现代沉积物中铁质 结核特征:对太湖形成机制的探讨. 沉积学报, 2014, 32(3): 503-509.]

[ 8 ] Zhang XZ, Wang YF, Lei HY. Authigenic mineralogy, depositional environments and evolution of fault-bounded lakes of the Yunnan Plateau, south-western China. Sedimentology, 1996, 43(2): 367-380.

[ 9 ] Safiur RM, Whalen M, Gagnon GA. Adsorption of dissolved organic matter (DOM) onto the synthetic iron pipe corrosion scales (goethite and magnetite): Effect of pH. Chemical Engineering Journal, 2013, 234 : 149-157.

[10] Lv J, Zhang S, Wang S et al. Molecular-scale investigation with ESI-FT-ICR-MS on fractionation of dissolved organic matter induced by adsorption on iron oxyhydroxides. Environmental Science \& Technology, 2016, 50(5) : 2328-2336.

[11] Qin X, Liu F, Wang G et al. Fractionation of humic acid upon adsorption to goethite: Batch and column studies. Chemical Engineering Journal, 2015, 269: 272-278.

[12] Huang CC, Li YM, Wang Q et al. Components optical property of CDOM in Lake Taihu based on three-dimensional excitation emission matrix fluorescence. J Lake Sci, 2010, 22(3) : 375-382. DOI: 10.18307/2010.0309. [黄昌春, 李云梅, 王桥等. 基于三维苂光和平行因子分析法的太湖水体 CDOM 组分光学特征. 湖泊科学, 2010, 22(3) : 375-382.]

[13] Sun W, Gong XL, Chen Y et al. Photochemical degradation of the algae-derived dissolved organic matter in Lake Taihu. $J$ Lake Sci, 2018, 30(1) : 91-101. DOI:10.18307/2018.0109. [孙伟, 巩小丽, 陈煜等. 太湖藻源溶解性有机质光化 学降解研究. 湖泊科学, $2018, \mathbf{3 0}$ (1): 91-101.]

[14] Yang L, Hur J. Critical evaluation of spectroscopic indices for organic matter source tracing via end member mixing analysis based on two contrasting sources. Water Research, 2014, 59: 80-89.

[15] Lee B, Seo Y, Hur J. Investigation of adsorptive fractionation of humic acid on graphene oxide using fluorescence EEM- 
PARAFAC. Water Research, 2015, 73: 242-251.

[16] Schwertmann U, Cornell RM. Iron oxides in the laboratory: Preparation and characterization. Weinheim: WILEYVCH, 2000.

[17] Liu X, Eusterhues K, Thieme J et al. STXM and NanoSIMS investigations on EPS fractions before and after adsorption to goethite. Environmental Science \& Technology, 2013, 47(7) : 3158-3166.

[18] Wang L, Liang N, Li H et al. Quantifying the dynamic fluorescence quenching of phenanthrene and ofloxacin by dissolved humic acids. Environmental Pollution, 2015, 196: 379-385.

[19] Hansen AM, Kraus TEC, Pellerin BA et al. Optical properties of dissolved organic matter (DOM) : Effects of biological and photolytic degradation. Limnology and Oceanography, 2016, 61(3) : 1015-1032.

[20] Murphy KR, Stedmon CA, Graeber D et al. Fluorescence spectroscopy and multi-way techniques. PARAFAC. Analytical Methods, 2013, 23(5) : 6557-6566.

[21] Wu P, Wen Y, Xiang Y et al. Sorption of pyrene by clay minerals coated with dissolved organic matter (DOM) from landfill leachate. Journal of Chemistry, 2015, 2015: 1-10.

[22] Gu B, Schmitt J, Chen Z et al. Adsorption and desorption of different organic matter fractions on iron oxide. Geochimica et Cosmochimica Acta, 1995, 59(2) : 219-229.

[23] Wu H, Lin Y, Wu J et al. A preliminary study of surface adsorption of iron oxide minerals for phenol and DOM. Earth Science Frontiers, 2008, 15(6) : 133-141.

[24] Xu H, Yu G, Yang L et al. Combination of two-dimensional correlation spectroscopy and parallel factor analysis to characterize the binding of heavy metals with DOM in lake sediments. Journal of Hazardous Materials, 2013, 263: 412-421.

[25] Cory RM, Kaplan LA. Biological lability of streamwater fluorescent dissolved organic matter. Limnology and Oceanography, 2012, 57(5) : 1347-1360.

[26] Saadi I, Borisover M, Armon R et al. Monitoring of effluent DOM biodegradation using fluorescence, UV and DOC measurements. Chemosphere, 2006, 63(3) : 530-539.

[27] Fu QL, He JZ, Blaney L et al. Roxarsone binding to soil-derived dissolved organic matter: Insights from multi-spectroscopic techniques. Chemosphere, 2016, 155: 225-233.

[28] Omoike A, Chorover J. Adsorption to goethite of extracellular polymeric substances from Bacillus subtilis. Geochimica et Cosmochimica Acta, 2006, 70(4) : 827-838.

[29] Vieira A P, Berndt G, de Souza Junior IG et al. Adsorption of cysteine on hematite, magnetite and ferrihydrite: FT-IR, Mössbauer, EPR spectroscopy and X-ray diffractometry studies. Amino Acids, 2011, 40(1) : 205-214.

[30] Lee BM, Hur J. Adsorption behavior of extracellular polymeric substances on graphene materials explored by fluorescence spectroscopy and two-dimensional fourier transform infrared correlation spectroscopy. Environmental Science \& Technology, 2016, 50(14) : 7364-7372.

[31] Noren K, Loring JS, Persson P. Adsorption of alpha amino acids at the water/goethite interface. Journal of Colloid and Interface Science, 2008, 319(2) : 416-428.

[32] Kaiser K, Zech W. Competitive sorption of dissolved organic matter fractions to soils and related mineral phases. Soil Science Society of America Journal, 1997, 61: 64-69.

[33] Romera-Castillo C, Chen M, Yamashita Y et al. Fluorescence characteristics of size-fractionated dissolved organic matter: implications for a molecular assembly based structure? Water Research, 2014, 55: 40-51.

[34] Liu J, Zhu RL, Xu TY et al. Interaction of polyhydroxy fullerenes with ferrihydrite: adsorption and aggregation. Journal of Environmental Sciences, 2018, 64:1-9.

[35] Gu B, Schmitt J, Chen Z et al. Adsorption and desorption of natural organic matter on iron oxide: mechanisms and models. Environmental Science \& Technology, 1994, 28(1) : 38-46.

[36] Bai LL, Cao CC, Wang CH et al. Roles of phytoplankton-and macrophyte-derived dissolved organic matter in sulfamethazine adsorption on goethite. Environmental Pollution, 2017, 230: 87-95. 